\title{
Adjacent Bi-level bilateral pedicle stress fractures after instrumented posterolateral lumbar fusion-a case report and review of the literature
}

\author{
João Pedro Jorge ${ }^{1} \cdot$ Nelson Carvalho $^{1}$
}

Received: 11 October 2018 / Accepted: 14 February 2019 / Published online: 19 February 2019

○) Springer-Verlag France SAS, part of Springer Nature 2019

\begin{abstract}
Isolated bilateral pedicle stress fractures of the lumbar spine are rare events, and few cases are reported in the literature. Their occurrence is commonly related to post-operative complications of spine instrumentation but can also be associated with stress-related activities, degenerative spine conditions, trauma and other miscellaneous causes. The authors report a case of adjacent bi-level bilateral pedicle fracture that developed 5 years after an instrumented posterolateral lumbar fusion. We believe that this has never been described before, and we reviewed the current literature pertaining this subject.
\end{abstract}

Keywords Pedicle fracture $\cdot$ Stress fracture $\cdot$ Low back pain $\cdot$ Neural arch $\cdot$ Spinal fusion

\section{Introduction}

Unlike spondylolysis, or stress fracture of the pars interarticularis, isolated bilateral pedicle stress fractures are extremely uncommon [1]. The biomechanical background of the pedicles, with increased strength and short moment arm than the pars, enables these structures to resist greater cyclic stress when force is transmitted through the vertebra [2]. These rare lesions can be related to trauma, activityrelated mechanical stress, altered bone biology or, most of the times, post-operative complications of spine instrumentation, mainly at the upper level of posterior fusion [3-5]. The authors report a case of bi-level adjacent bilateral pedicle fracture after a posterolateral L4-L5 fusion, which, to the best of our knowledge, has never been described, and we provide an explanation to this event in light with the current literature.

João Pedro Jorge

joaopljjorge@gmail.com

1 Centro Hospitalar Universitário de Lisboa Central Hospital de Curry Cabral, Rua da Beneficência, $n^{\circ} 8$, 1069-166 Lisbon, Portugal

\section{Case report}

An 87-year-old woman was sent to our consultation office due to unrelenting back pain with 3 months of evolution, which started spontaneously after standing up from a chair, without any history of trauma or stress-related activity. Five years previously to this episode, she had L4-L5 posterolateral fusion with cement-augmented transpedicular screws under a diagnosis of lumbar spine stenosis, in another institution. Physical examination revealed no motor weakness or sensory changes, but significant tenderness was present in the low back area and motion was limited, especially extension. Plain radiographs were without significant alterations - the instrumentation was adequately placed, and there were no signs of fracture or instability on the flexion/extension X-rays (Fig. 1). Adjacent segment disease with decreased intervertebral space and sclerosis of the upper plate of L4 was evident. Computed tomography (CT) revealed bi-level bilateral pedicle fractures through L2 and L3 (Fig. 2). We performed an extension of the posterior fixation with transpedicular screws in L2 and L3 (Fig. 3). Follow-up of 3 months revealed no residual low back pain and the patient returned to normal daily activities. 
Fig. 1 Above - AP and lateral view of the lumbar spine - No signs of fracture or failure of instrumentation. Below - Flexion/extension x-rays - no signs of instability

Fig. 2 Sagittal and axial CT cuts revealed bi-level bilateral pedicle fractures through L2 and L3
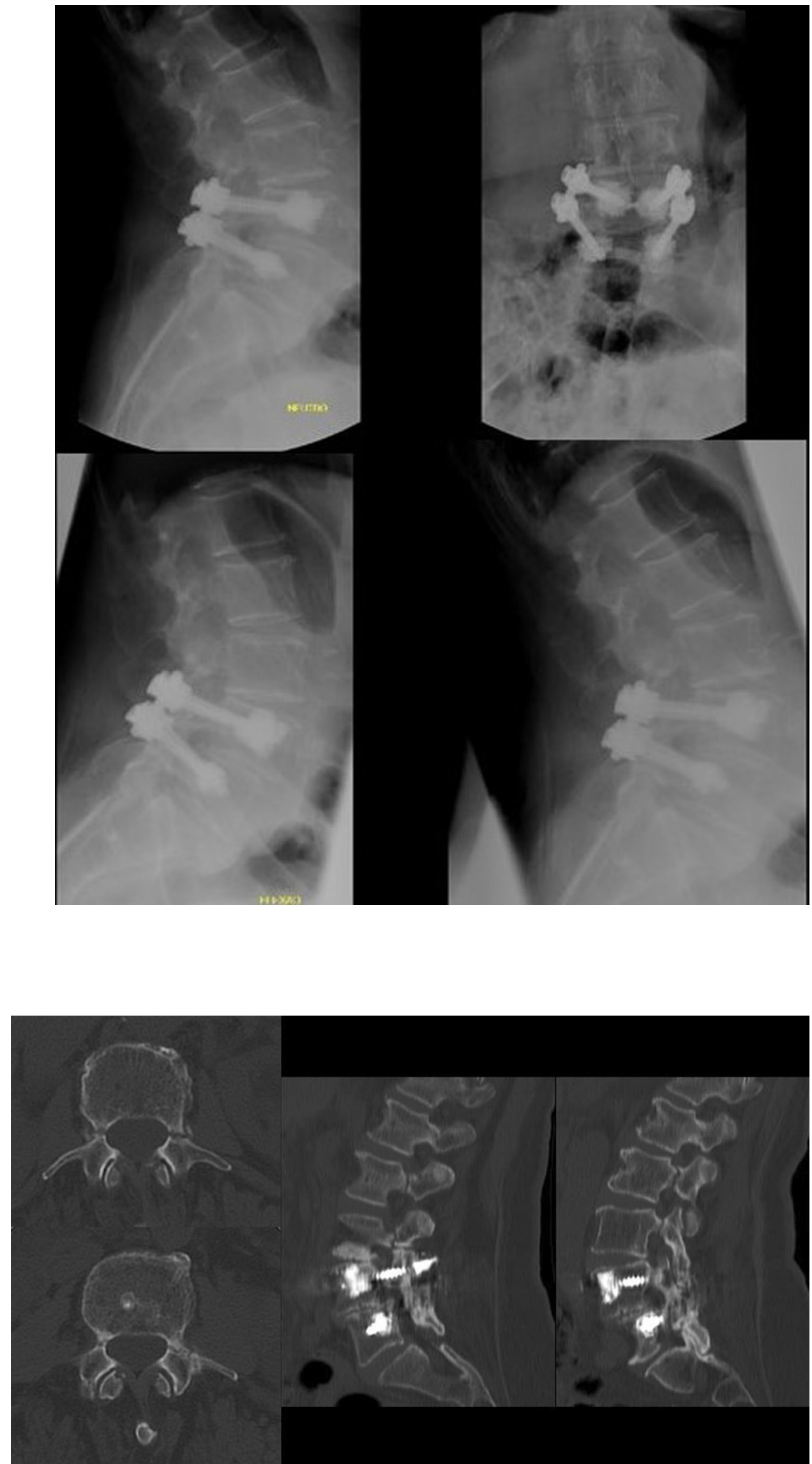


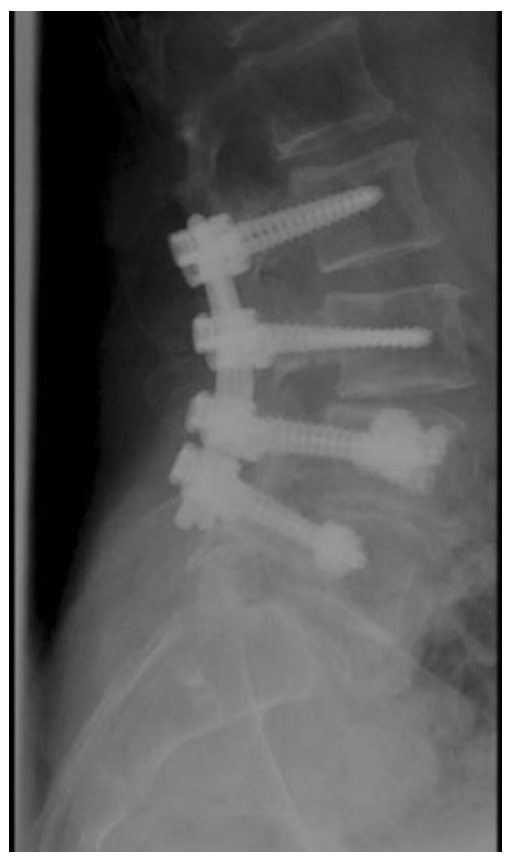

Fig. 3 Extension of the posterior fixation with transpedicular screws in L2 and L3

\section{Discussion}

The first reported case of bilateral pedicle stress fracture dates from 1991 [6]. Stress fractures occur in normal or abnormal bone when repetitive mechanical load exceeds the biological capacity of the bone [4]. Depending on the amount of stress applied to bone and on the elastic properties of bone tissue, they are classified as fatigue or insufficiency fractures. Fatigue fractures occur when abnormal muscular stress or torque is applied to bone with normal elastic resistance. Insufficiency fractures occur when normal physiological muscular stress is applied to bone with deficient elastic resistance or mineral content $[7,8]$. The spine is a common fracture site secondary to osteoporosis, specifically, vertebral body compression fractures. However, stress fractures of the pedicle are much less common [9]. Vertebral pedicles are part of the posterior neural arch, and, like the pars interarticularis, are vulnerable to cyclic loads, although fractures of the former occur much more rarely than of the latter [10], mainly due to the fact that the pedicle has greater intrinsic strength and a shorter moment arm from the vertebral body and therefore can resist greater cyclic shear forces [11]. Cyron et al. [12] reported 5 pedicle stress fractures compared to 53 pars interarticularis fractures in vertebras subjected to experimental cyclic shear loads.

Unilateral pedicle stress fracture has been clearly described in the literature and is commonly associated with contralateral spondylolysis [13-16]. On the other hand, bilateral pedicle stress fracture is an uncommon event and has been mostly associated with previous spine surgery [2, 17-20] and activity-related mechanical stress [11, 21, 22], although there are other case reports of bilateral pedicle stress fractures without the above-mentioned aetiologiesKim et al. [23] reported the case of a bilateral pedicle stress fracture on the second lumbar vertebra with spondylolisthesis in a 63-year-old female patient in the setting of an ankylosing spondylitis; Sadiq [9] reported the case of a bilateral pedicle stress fractures of the second lumbar vertebra in a 36-year-old patient with a sedentary lifestyle and with no risk factor or inciting event; Han et al. [10] also reported a bilateral pedicle fracture of L5 on a 50-year-old female without an inciting event or cause; Doita et al. [7] reported 2 cases of bilateral pedicle stress fractures. The first case was a 57-year-old male patient with a lumbar canal stenosis and bilateral pedicle stress fracture on L4. The second case was a 77 -year-old female patient with an L5 vertebral body osteoporotic fracture with bilateral pedicle stress fracture on L4; Kang et al. [24] also reported a case of bilateral pedicle fracture of L3 in the setting of osteoporotic vertebral compression fractures of L2 and L4; Hajjioui et al. [5] reported the clinical case of a 54-year-old woman with L4-L5 spondylolisthesis due to bilateral pedicle fracture in the setting of steroid-induced osteoporosis for benign rheumatoid polyarthritis; Karabay et al. [4] reported a fourth lumbar bilateral pedicle fracture in a patient with osteoporosis and bisphosphonate therapy; and El Rachkidi at. al [25] reported a bilateral L5 pedicle fracture due to an 10 years usage of bisphosphonate therapy.

Load distribution is absorbed mainly by the intervertebral disc in the anterior segment. In the posterior segment, the load passes through the facet joints and is distributed among the posterior elements (pars interarticularis, laminae, and pedicles), the pars being the most susceptible to injury, followed by the pedicles [26]. A stress fracture of the lumbar pedicle is rare but can occur, especially in conditions in which the load on the neural arch is abnormally distributed. One setting in which the pedicle can experience increased stress is after surgery involving the posterior elements, whether after decompression surgery [27-29] or in the setting of posterolateral fusion with pedicle screws $[2,17-19,30]$. It is theorized that pedicles associated with posterolateral fusion masses experience abnormal repetitive shear forces due to continued motion through intervertebral discs, and the pars interarticularis, which normally shares these forces, is fixed in the fusion mass [21, 31].

Our patient did not have any predisposing risk factors for pedicle fracture except severe age-derived osteoporosis (which explains the usage of cemented screws on the first surgery). Osteoporotic bone loss resulting in material and structural changes of the bone leads to an increased risk of stress fractures due to the weaken structure of the spinal bones [3]. Also, osteoporosis contributes to 
cephalad compression fractures due to altered biomechanics in adjacent segments associated with loss of motion at fused segments [32]. The rise in the stress concentration of the proximal spine segments leads to severe junctional changes. These acquired disturbances of the neural arch cause abnormal repetitive shear and cantilever forces, which result most of the times in fractures of the weakest zone, the pars interarticularis (spondylosis) or, in rarest occasions and as in our case report, fracture of the pedicles $[19,32]$. Junctional pedicles, rather than the pedicles of the upper instrumented vertebra, are the ones that experience repetitive shear forces, because the latter are within the fusion mass. This is the physiopathology premise of our clinical case-with a stiff fusion mass at the lower lumbar spine augmented in strength due to the use of cement on the pedicle screws, a stress rise effect on the upper lumbar segments led to a combination of fatigue and insufficiency fracture of the pedicles. Only 1 very recent (2017) paper [32] reports adjacent bilateral pedicle fractures after instrumented lumbar fusion. To our knowledge, there is no report on a bi-level adjacent bilateral pedicle fractures after instrumented lumbar fusion.

Due to the maintenance of pain despite conservative treatment, surgical intervention was performed, and the authors decided to extend the posterior fusion to the injured levels, with transpedicular screws at L2 and L3 levels. The use of transpedicular screws may, in a theoretical basis, delay fracture healing, as it disturbs the blood supply to the fracture site [31]. The literature does not show any evidence that a pedicle screw causes avascular necrosis or delayed fracture healing, so uneventful healing was expect to occur. At 3-month follow-up, our patient was pain-free (VAS 0). The X-ray showed evidence of healing of both fracture lines. The patient resumed daily activities without pain.

\section{Conclusion}

Pedicle stress fracture adjacent to an instrumented fusion mass is an uncommon entity, and a bi-level pedicle stress fracture has never been reported before. It may, however, be a source of symptoms in patients with back pain after spine fusion. Surgeons caring for this group of patients should be aware of this condition.

\section{Compliance with ethical standards}

Conflict of interest The authors declare that they have no conflict of interest.

\section{References}

1. Maruo K, Tachibana T, Inoue S, Arizumi F, Yoshiya S (2015) Unilateral pedicle stress fracture in a long-term hemodialysis patient with isthmic spondylolisthesis. Case Rep Orthop 2015:426940

2. Robertson PA, Grobler LJ (1993) Stress fracture of the pedicle. A late complication of posterolateral lumbar fusion. Spine (Phila Pa 1976) 18:930-932

3. Kim HS, Kim SW, Lee WT (2012) Spondylolisthesis accompanying bilateral pedicle stress fracture at two vertebrae. J Korean Neurosurg Soc 51:388-390

4. Karabay N, Ozer E, Ada E (2015) Multi-level, bilateral pedicle fractures: case report. Turk Neurosurg 25(2):340-343

5. Hajjioui A, Khazzani H, Sbihi S, Bahiri R, Benchekroune B, Hajjaj-Hassouni N (2011) Spondylolisthesis on bilateral pedicle stress fracture in the lumbar spine: a case study. Ann Phys Rehabil Med 54:53-58

6. Traughber PD, Havlina JM Jr (1991) Bilateral pedicle stress fractures: SPECT and CT features. J Comput Assist Tomogr 15:338-340

7. Doita M, Ando Y, Hirata S, Ishikawa H, Kurosaka M (2009) Bilateral pedicle stress fracture in a patient with osteoporotic compression fracture. Eur Spine J 18(Suppl 2):206-209

8. Schaffler MB, Radin EL, Burr DB (1990) Long-term fatigue behaviour of compact bone at low strain magnitude and rate. Bone 11:321-326

9. Sadiq MZ (2006) Bilateral pedicle stress fracture in the lumbar spine of a sedentary office worker. Eur Spine J 15(Suppl. 5):S653-S655

10. Han SH, Hyun SJ, Jahng TA, Kim KJ (2016) Posterior osteosynthesis of a spontaneous bilateral pedicle fracture of the lumbar spine. J Neurosurg Spine 24(3):398-401

11. Gunzburg R, Fraser RD (1991) Stress fracture of the lumbar pedicle Case reports of "pediculolysis" and review of the literature. Spine (Phila Pa 1976) 16:185-189

12. Cyron BM, Hutton WC (1978) The fatigue strength of the lumbar neural arch in spondylolysis. J Bone Joint Surg Br $60-\mathrm{B}(2): 234-238$

13. Aland C, Rineberg BA, Malberg M et al (1986) Fracture of the pedicle of the fourth lumbar vertebra associated with contralateral spondylolysis. Report of a case. J Bone Joint Surg Am 68:1454-1455

14. Garber JE, Wright AM (1986) Unilateral spondylolysis and contralateral pedicle fracture. Spine 11:63-66

15. Maurer SG, Wright KE, Bendo JA (2000) Iatrogenic spondylolysis leading to contralateral pedicular stress fracture and unstable spondylolisthesis: a case report. Spine 25:895-898

16. Schmid T, Heini P, Benneker L (2017) A rare case of non-traumatic, multi-level, bilateral pedicle fractures of the lumbar spine in a 60-year-old patient. Eur Spine J 26(Suppl 1):197-201

17. Knight RQ, Chan DP (1992) Idiopathic scoliosis with unusual stress fracture of the pedicle within solid fusion mass. A case report. Spine 17:849-851

18. Macdessi SJ, Leong AK, Bentivoglio JE (2001) Pedicle fracture after instrumented posterolateral lumbar fusion: a case report. Spine 26:580-582

19. Tribus CB, Bradford DS (1993) Bilateral pedicular stress fractures after successful posterior spinal fusion for adult idiopathic scoliosis. Spine 18:1222-1225

20. Lattig F, Fekete TF, Jeszenszky D (2010) Management of fractures of the pedicle after instrumentation with transpedicular screws: a report of three patients. J Bone Joint Surg Br 92(1):98-102

21. Parvataneni HK, Nicholas SJ, McCance SE (2004) Bilateral pedicle stress fractures in a female athlete: case report and review of the literature. Spine 29:E19-E21 
22. Amari R, Sakai T, Katoh S, Sairyo K, Higashino K, Tachibana $\mathrm{K}$, Yasui N (2009) Fresh stress fractures of lumbar pedicles in an adolescent male ballet dancer: case report and literature review. Arch Orthop Trauma Surg 129(3):397-401

23. Kim HS, Ju CI, Kim SW (2010) Bilateral pedicle stress fracture accompanying spondylolysis in a patient with ankylosing spondylitis. J Korean Neurosurg Soc 48:70-72

24. Kang HJ, Hong JY, Park JW, Suh SW (2014) Bilateral pedicle stress fracture with adjacent old osteoporotic compression fractures that induced spondylolisthesis. J Orthop Sci 19(4):682-685

25. El Rachkidi RL, Sari-Leret ML, Wolff S (2011) Atypical bilateral pedicle fracture in long-term bisphosphonate therapy. Spine (Phila Pa 1976) 36(26):E1769-E1773

26. Estrada JA, Fernandez JR, Garnier JC, Johnson MD, Lopez FC (2014) Bilateral fracture of L5 pedicles in a patient with total disc replacement of L5-S1: a case report. Coluna/Columna 13(2):153-155

27. Sirvanci M, Ulusoy L, Duran C (2002) Pedicular stress fracture in lumbar spine. Clin Imaging 26(3):187-193

28. Stanley D, Smith TW (1990) Contralateral pedicle stress fracture. An unusual complication of laminectomy. Spine (Phila Pa 1976) 15(6):598-599
29. Sheehan JP, Helm GA, Sheehan JM, Jane JA Sr (2002) Stress fracture of the pedicle after extensive decompression and contralateral posterior fusion for lumbar stenosis. Report of three cases. Neurosurg Focus 13(2):E9

30. Ha KY, Kim YH (2003) Bilateral pedicle stress fracture after instrumented posterolateral lumbar fusion: a case report. Spine 28:E158-E160

31. Johnson JN, Wang MY (2009) Stress fracture of the lumbar pedicle bilaterally: surgical repair using a percutaneous minimally invasive technique. J Neurosurg Spine 11(6):724-728

32. Kim HS, Ha SW, Ju CI, Kim SW (2017) Adjacent bilateral stress pedicle fractures after instrumented lumbar fusion: a case report. Korean J Neurotrauma 13(1):54-56

Publisher's Note Springer Nature remains neutral with regard to jurisdictional claims in published maps and institutional affiliations. 